\title{
DOR SOFRIDA PELO RECÉM-NASCIDO DURANTE A PUNÇÃO ARTERIAL ${ }^{a}$

\author{
Pain in the newborn during the arterial puncture \\ Dolor sufrido por el recién nacido durante la punción arterial
}

\section{RESUMO}

Objetivou-se avaliar a intensidade da dor sofrida pelo recém-nascido prematuro na Unidade de Terapia Intensiva Neonatal, durante a coleta de sangue arterial, por intermédio da Neonatal Infant Pain Scale (NIPS), utilizando medidas de sucção não nutritiva. Estudo de intervenção, quantitativo, realizado com 24 recém-nascidos em Fortaleza-CE, em 2004. 0 grupo-caso recebeu uma chupeta de gaze embebida em água destilada, e o grupo-controle, uma chupeta com glicose a $25 \%$, dois minutos antes da coleta. $\mathrm{Na}$ análise inferencial, a intensidade média da dor com água destilada foi 6,08; com glicose a $25 \%$ foi de 1,04. Concluímos que as alterações comportamentais variaram significativamente, já as fisiológicas pouco foram alteradas.

Palavras-chave: Dor. Recém-nascido. Enfermagem. Neonatologia.

\begin{abstract}
The purpose of this study was to evaluate the pain intensity in the premature newborn hospitalized in the Neonatal Intensive Care Unit through Neonatal Infant Pain Scale (NIPS), during the arterial blood collect using non nutritious suction. It was a quantitative, intervention study accomplished with 24 newborns in Fortaleza - Ceará, in 2004. The experimental group received a gauze pacifier soaked in distilled water and the control group received a gauze pacifier soaked in $25 \%$ of glucose, both used two minutes before the blood collect. In the inferential analysis the average intensity of the pain with the group of distilled water was 6,08 while with the group of $25 \%$ of glucose the average was 1,04 . We then concluded that the behavior changes showed intense modifications, while the physiological ones didn't present meaningful variations.
\end{abstract}

Key words: Pain. Newborn. Nursing. Neonatology.

\section{Resumen}

El objetivo fue evaluar la intensidad del dolor en el recién nacido prematuro en la Unidad de Terapia Intensiva Neonatal durante la toma de sangre arterial, a través de la Escala neonatal de dolor infantil ( Neonatal Infant Pain Scale (NIPS), utilizándose medidas de succión no nutritiva. Estudio de intervención, cuantitativo, realizado con 24 Recién nacidos en Fortaleza-CE, en 2004. El grupo caso recibió un chupón de gasa embebido en agua destilada, y el grupo control un chupón con una solución glucosada al $25 \%$, dos minutos antes de la toma. En el análisis inferencial, la intensidad media del dolor en el grupo con agua destilada fue de 6.08 , y en el grupo con la solución glucosada al $25 \%$ fue de 1.04 . Concluimos que las alteraciones comportamentales variaron significativamente, pues las fisiológicas registraron pocos cambios.

Palabras Clave: Dolor. Recién Nacido. Enfermería. Neonatología.

'Enfermeira assistencial. Especialista em Enfermagem Neonatal. Brasil. E-mail: terezamonica@hotmail.com, ${ }^{2}$ Enfermeira. Mestre em Saúde da Criança e do Adolescente. Doutoranda em Farmacologia/UFC. Membro do projeto de pesquisa Saúde do Binômio Mãe-Filho/UFC. Brasil. E-mail: ednacam3@hotmail.com, ${ }^{3}$ Enfermeira. Doutora em Enfermagem. Bolsista de Produtividade 2 CNPq. Profa. Adjunta do Departamento de Enfermagem /UFC. Coordenadora do Projeto de Pesquisa Saúde do Binômio Mãe-Filho/UFC. Brasil. E-mail: cardoso@ufc.br 


\section{INTRODUCÃO}

0 avanço tecnológico para deteç̧ão de problemas relacionados aos recém-nascidos (RNs) e o incremento de recursos terapêuticos disponíveis atualmente propiciam 0 aumento da sobrevida dessas crianças. Paralelamente a isto, observa-se o crescente número de manuseios e procedimentos, sendo na maioria das vezes desnecessários e agressivos, ao ponto de causarem dor, podendo alterar e desestruturar todo 0 sistema orgânico do RN.

Durante as primeiras horas de vida, os RNs prematuros e de risco são submetidos a vários procedimentos dolorosos, tais como: intubação, aspiração da cânula orotraqueal, coleta de exames através da punção arterial, acesso venoso, drenagem de tórax etc. A gasometria arterial (GA) é um exame considerado fundamental e rotineiro em Unidade de Terapia Intensiva Neonatal (UTIN) para os recém-nascidos que experimentam desordens respiratórias, visto que a principal função do sistema respiratório é realizar as trocas gasosas na respiração (oxigênio e dióxido de carbono).

Acredita-se que todos os RN que se encontram internados na UTIN recebam, em média, cerca de 50 a 150 procedimentos potencialmente dolorosos e que em RNs com menos de 1.000 gramas esse número chega a 500 ao longo de sua internação. ${ }^{1}$

No momento atual, uma atenção especial à dor e a busca de meios para diminui-la e até evitá-la podem ser um passo importante para a melhoria da qualidade de vida do RN internado em UTIN. Almejando uma atenção mais humanizada durante os procedimentos dolorosos, pode-se passar a intervir de forma vigorosa, de modo a atenuar esse sofrimento, minimizando as consequências da dor.

A avaliação da dor no recém-nascido torna-se um desafio. Para quantificação e qualificação da dor, têm-se utilizado escalas que levam em conta alterações comportamentais e fisiológicas no período neonatal. Entre as escalas utilizadas para avaliação da dor no recém-nascido, citam-se: Neonatal Infant Pain Scale (NIPS), Sistema de Codificação da Atividade Facial Neonatal (NFCS) desenvolvida por GRUNAU e CAIG, e Premature Infant Pain Profile (PIPP). 2,3,4

A equipe de enfermagem pode aplicar a escala Neonatal Infant Pain Scale (NIPS) a todo RN internado em UTIN simultaneamente à realização dos sinais vitais; em uma escala cuja pontuação varia de zero a sete pontos, pontuações superiores a três devem alertar para a necessidade de introdução ou adequação da dose de analgésicos. ${ }^{4}$

0 interesse pelo tema surgiu de nossa prática cotidiana na UTIN, quando observamos a não utilização de estratégias de minimização da dor durante os procedimentos dolorosos com 0 RN. Reconhecer e caracterizar o sinal de dor deve ser um compromisso do enfermeiro que presta assistência ao $\mathrm{RN}$ em estado grave.

Pesquisar especificamente a dor na punção arterial se faz relevante, visto ser um procedimento rotineiro no âmbito das UTINs, que exige conhecimento e habilidade na sua execução.
Diante do exposto, utilizou-se uma escala para aferir a dor durante a punção arterial no recém-nascido, sendo objetivo deste estudo avaliar a dor sofrida pelo recém-nascido prematuro internado em UTIN durante a punção arterial periférica realizada pela enfermeira, utilizando-se como instrumento a escala de dor Neonatal Infant Pain Scale - NIPS.

\section{MÉTODO}

Trata-se de um estudo de intervenção, quantitativo, no qual os participantes foram tomados de forma não probabilística para compor o grupo do estudo. Foi realizada em uma UTIN da rede pública de Fortaleza, credenciada ao Sistema Único de Saúde, composta por 25 leitos, dos quais 12 são destinados à assistência intensiva e 13, à semi-intensiva.

A população foi composta por 32 RNs que foram admitidos no período da coleta de dados; a amostra foi do tipo intencional, totalizando 24 RNs que se encontravam internados na UTIN em suporte ventilatório, com controle dos gases sanguíneos mediante gasometria arterial no período de setembro a outubro de 2004

As variáveis para a caracterização da amostra foram: peso ao nascimento, sexo, idade gestacional e diagnóstico médico. $\mathrm{A}$ variável independente foi o uso da chupeta de gaze embebida em glicose a 25\%. A variável dependente foi a ocorrência de alterações comportamentais e fisiológicas durante a coleta de sangue arterial.

0 grupo foi composto por 24 recém-nascidos, e cada um foi seu próprio controle. 0 paciente pode ser usado como seu controle em intervenções com a finalidade de estudar o efeito de tratamentos de aplicação tópica ${ }^{5}$. Nesses casos, cada pessoa pertence tanto ao grupo experimental como ao grupo-controle, ao mesmo tempo. Para garantir a validade do experimento, é preciso garantir, que nenhum outro fator, além da intervenção, agiu sobre o indivíduo.

0 grupo-caso recebeu uma chupeta de gaze embebida com solução de água destilada (AD) dois minutos antes da coleta do sangue arterial, enquanto o grupo-controle recebeu uma chupeta de gaze embebida em glicose $25 \%$ dois minutos antes da coleta de sangue arterial. 0 RN foi mantido com oxímetro de pulso para registro da frequência de pulso e saturação de oxigênio. 0 primeiro registro ocorreu um minuto antes da coleta da gasometria arterial. 0 segundo aconteceu durante a realização do procedimento. As anotações foram realizadas por uma das pesquisadoras.

Foi utilizado um formulário contendo os dados de identificação do RN, indicadores de monitoração observados pelo oxímetro de pulso e os parâmetros da escala de avaliação de dor - a Neonatal Infant Pain Scale - NIPS - composta por seis indicadores de dor, sendo cinco comportamentais e um fisiológico, a saber Expressão facial (0 ou 1 ponto); Choro ( 0 ou 1 ou 2 pontos); Posição das pernas (0 ou 1 ponto); Respiração (0 ou 1 ponto); Posição dos braços (0 ou 1 ponto); 
Estado de sono ou vigília (0 ou 1 ponto). Se a pontuação final for superior a três pontos, considera-se a dor presente. ${ }^{6}$

Os dados foram analisados por meio do programa SPSS 13.0 (Statistical Package for Social Sciences) e apresentados em gráficos e tabelas e à luz da literatura pertinente. Para análise inferencial, foi empregado o t de Student para amostras pareadas, o r de Pearson e um nível de significância de $99 \%$ $(p=0,01)$.

Quanto aos aspectos éticos, a pesquisa foi aprovada pelo Comitê de Ética em Pesquisa do Hospital onde foi realizada, conforme Resolução 196/96, sobre pesquisa que envolve seres humanos, do Conselho Nacional de Saúde/ Ministério da Saúde, com registro no CEP: 09020904. Os pais/responsáveis pelo RN assinaram um termo de consentimento pós-esclarecido autorizando a participação dos mesmos na pesquisa.

\section{APRESENTAÇÃO E ANÁLISE DOS RESULTADOS}

No período do estudo, 32 RNs foram internados na UTIN. Destes, 24 participaram do estudo. Os dados de identificação contidos no instrumento de pesquisa foram relevantes para se conhecer o perfil dos RNs estudados, juntamente com os dados da idade gestacional dos RNs a fim de comprovar que, independentemente da imaturidade do sistema orgânico do recém-nascido pré-termo (RNPT), este já apresenta substratos anatômicos capazes de transmitir a dor.

Quanto ao perfil dos RNs, a maioria é do sexo masculino (16 RNs), e a minoria, feminina (8 RNs). Foram encontrados RNs com idade gestacional máxima de 37 semanas e mínima de 27 semanas, sendo a média 31,5 semanas.

Em relação ao peso ao nascer, o valor máximo foi de 3.465 $\mathrm{g}$, e o valor mínimo foi de $620 \mathrm{~g}$, sendo a média de $2.042 \mathrm{~g}$. De acordo com os dados, o diagnóstico mais frequente foi a síndrome do desconforto respiratório (SDR) associada à prematuridade com 12 (50\%), seguida da prematuridade extrema com 06 (25\%), SDR (04\%) e aspiração meconial e hérnia diafragmática ambos com 1 caso, (4\%).

De acordo com esses dados, a maior frequência de internação na UTIN relaciona-se a prematuridade, que pode vir associada a outras patologias ou não. Assistência terapêutica ao RN com as inovações tecnológicos, bem como o diagnóstico precoce tem contribuído para o aumento da sobrevida dos RNPT proporcionando diminuição da morbimortalidade dos RNs gravemente enfermos. ${ }^{7}$ Em contrapartida, eleva-se o número de exames e procedimentos invasivos para esclarecimentos diagnósticos, com custo elevado que inclui a dor. ${ }^{8}$

Tabela 1. Análise descritiva geral da amostra quanto à intensidade da dor antes e durante o uso da água destilada e glicose a 25\%. Fortaleza - CE. ago/set. 2004.

\begin{tabular}{|c|c|c|c|c|c|c|c|}
\hline \multirow[t]{2}{*}{$\begin{array}{c}\text { Medidas de sucção não } \\
\text { nutritivas }\end{array}$} & \multicolumn{2}{|c|}{ Variáveis } & \multirow{2}{*}{$\begin{array}{l}N \\
24\end{array}$} & \multirow{2}{*}{$\begin{array}{c}\text { Mínimo } \\
27\end{array}$} & \multirow{2}{*}{$\begin{array}{c}\text { Máximo } \\
37\end{array}$} & \multirow{2}{*}{$\begin{array}{l}\text { Média } \\
33,04\end{array}$} & \multirow{2}{*}{$\begin{array}{l}\begin{array}{l}\text { Desvio- } \\
\text { padrão }\end{array} \\
2,48\end{array}$} \\
\hline & $\overline{I G C}$ & rigida & & & & & \\
\hline \multirow{5}{*}{ Água destilada } & Intensid & de da dor & 24 & 01 & 07 & 6,08 & 1,35 \\
\hline & $\mathrm{FP}$ & Antes & 24 & 103 & 165 & 129,38 & 19,06 \\
\hline & $(\mathrm{bpm})$ & Durante & 24 & 68 & 184 & 156,83 & 23,85 \\
\hline & $\mathrm{SO}_{2}(\%)$ & Antes & 24 & 92 & 98 & 96,13 & 1,87 \\
\hline & & Durante & 24 & 74 & 99 & 90,96 & 6,32 \\
\hline \multirow[t]{4}{*}{ Glicose 25\% } & Intensid & de da dor & 24 & 0 & 06 & 1,04 & 1,60 \\
\hline & $\mathrm{FP}(\mathrm{bpm})$ & Antes & 24 & 98 & 170 & 130,63 & 19,20 \\
\hline & & Durante & 24 & 96 & 182 & 137,38 & 20,86 \\
\hline & $\mathrm{SO}_{2}(\%)$ & $\begin{array}{c}\text { Antes } \\
\text { Durante }\end{array}$ & $\begin{array}{l}24 \\
24\end{array}$ & $\begin{array}{l}89 \\
89\end{array}$ & $\begin{array}{l}99 \\
98\end{array}$ & $\begin{array}{l}95,50 \\
95,29\end{array}$ & $\begin{array}{l}2,75 \\
2,60\end{array}$ \\
\hline
\end{tabular}

De acordo com a Tabela 1, a intensidade da dor teve como valor máximo 7 escores, e mínimo 1 escore, média de 6,08, com o uso de água destilada; já com o uso de glicose a 25\%, 0 valor máximo foi de 6 e mínimo, de zero escore, média de 1,04 .

Os escores relacionados à dor, com o uso de chupeta embebida com glicose a $25 \%$, foram encontrados no máximo de 6 pontos em um caso isolado, o qual se enquadrava na categoria dos RNs com incapacidade de sucção da chupeta por ter idade gestacional menor do que 29 semanas.
Quanto à frequência de pulso (FP) registrada antes do procedimento doloroso com o uso de água destilada, o valor máximo foi 165 bpm, e o mínimo foi de 103 bpm, média de 129,38. Durante o procedimento, a FP máxima foi de 186 bpm e a mínima de 68 bpm, com média de 156,83bpm. Em relação à saturação de oxigênio $\left(\mathrm{SO}_{2}\right)$, nas mesmas condições antes do procedimento doloroso, o máximo foi de $98 \%$ e o mínimo foi de $92 \%$, com média de 96,13\%. Durante o procedimento, o máximo foi de $99 \%$ e o mínimo de $76 \%$, com média de $90,96 \%$. 
As variáveis $\mathrm{FC}$ e $\mathrm{SaO}_{2}$ são parâmetros que não podem ser avaliados isoladamente como presença de dor. Em geral, após - estímulo nociceptivo, o RN apresenta elevação de FC e queda de $\mathrm{SaO}_{2}$ em termos absolutos. Estes são úteis para avaliação da dor, pois são medidas fáceis e práticas, porém, não são medidas específicas de dor, pois sua modificação não ocorre somente diante de estímulos dolorosos, mas também na vigência de estímulos desagradáveis e/ou estressantes. ${ }^{4}$

Tabela 2. Análise descritiva da amostra conforme intensidade da dor de acordo com a NIPS. Fortaleza - CE - ago/set. 2004.

\begin{tabular}{|c|c|c|c|c|c|c|c|c|c|c|c|c|}
\hline \multirow{3}{*}{$\begin{array}{c}\text { Medidas de sucção } \\
\text { não nutritivas }\end{array}$} & \multirow{2}{*}{\multicolumn{2}{|c|}{ Variáveis }} & \multicolumn{10}{|c|}{ Intensidade da dor } \\
\hline & & & \multicolumn{5}{|c|}{ Suportável $(\leq 3)$} & \multicolumn{5}{|c|}{ Intensa ( > 3 ) } \\
\hline & & & $N$ & Mín. & Máx & Média & $\begin{array}{l}\text { Desvio- } \\
\text { padrão }\end{array}$ & $N$ & Mín. & Máx & Média & $\begin{array}{l}\text { Desvio- } \\
\text { padrão }\end{array}$ \\
\hline \multirow{6}{*}{$\begin{array}{c}\text { Água } \\
\text { destilada }\end{array}$} & $\mathrm{FP}$ & Antes & 01 & 136 & 136 & 136,00 & - & 23 & 103 & 165 & 129,09 & 19,44 \\
\hline & (bpm) & Durante & 01 & 139 & 139 & 139,00 & - & 23 & 68 & 184 & 157,61 & 24,07 \\
\hline & $\mathrm{SO}_{2}(\%)$ & Antes & 01 & 98 & 98 & 98,00 & - & 23 & 92 & 98 & 96,04 & 1,87 \\
\hline & & Durante & 01 & 98 & 98 & 98,00 & - & 23 & 74 & 99 & 90,65 & 6,28 \\
\hline & FP & Antes & 21 & 98 & 170 & 129,57 & 18,15 & 03 & 113 & 170 & 138,00 & 29,14 \\
\hline & $(\mathrm{bpm})$ & Durante & 21 & 96 & 174 & 136,38 & 19,36 & 03 & 115 & 182 & 144,33 & 34,27 \\
\hline \multirow{2}{*}{ Glicose 25\% } & $\mathrm{SO}_{2}(\%)$ & Antes & 21 & 89 & 99 & 95,38 & 2,85 & 03 & 94 & 98 & 96,33 & 2,08 \\
\hline & & Durante & 21 & 89 & 98 & 95,38 & 2,58 & 03 & 91 & 97 & 94,67 & 3,21 \\
\hline
\end{tabular}

A Tabela 2 relaciona as variáveis alterações fisiológicas ( $F P$ e $\mathrm{SO}_{2}$ ), tanto com AD como com glicose $50 \%$, com a intensidade da dor, suportável, com escore d" 3 , ou intensa, escore $>3$.

Com o uso de $\mathrm{AD}$, a dor considerada suportável foi registrada em apenas um RN, que teve como alterações na FP: antes do procedimento doloroso, máxima, mínima e média de 136 bpm; durante o procedimento, máxima, mínima e média de 139 bpm. E alterações de $\mathrm{SO}_{2}$ : antes e durante o procedimento doloroso a máxima, mínima e média mantiveram-se em $98 \%$. Este caso isolado justifica-se, pois o RN encontrava-se em estado comatoso.

Já com a água destilada com a dor considerada intensa, foram obtidos 23 RNs que tiveram como alterações de FP: antes do procedimento doloroso, máxima 165 bpm, mínima 103 bpm e média de 126,09 bpm; durante o procedimento, máxima 184 bpm, mínima 68 bpm e média de 157,61bpm. E alterações de $\mathrm{SO}_{2:}$ antes do procedimento, máxima $98 \%$, mínima $92 \%$ e média de $96,04 \%$; e durante o procedimento máxima $99 \%$, mínima $74 \%$ e média de $90,65 \%$.

Em relação ao uso de glicose a 25\%, a dor considerada suportável foi registrada em 21 RNs que tiveram como alterações de FP: antes do procedimento doloroso, máxima 170 bpm, mínima 98 bpm e média de 129,57bpm; durante 0 procedimento, máxima 174bpm, mínima 96 bpm e média de 136,38 bpm. E alterações de $\mathrm{SO}_{2:}$ antes do procedimento doloroso, máxima $99 \%$, mínima $89 \%$ e média $95,38 \%$; durante, máxima 98\%, mínima $89 \%$ e média de $95,38 \%$.
Com o uso de glicose a $25 \%$, na dor considerada intensa, foram obtidos 3 RNs que tiveram alterações de FP: antes do procedimento doloroso, máxima 170 bpm, mínima 113 bpm e média de 138 bpm; durante o procedimento, máxima 182 bpm, mínima 115 bpm e média de 144,33bpm. E alterações de $\mathrm{SO}_{2}$ : antes do procedimento, máxima 98\%, mínima $94 \%$ e média de $96,33 \%$; e durante o procedimento máxima $97 \%$, mínima $91 \%$ e média de $94,67 \%$.

Em relação aos RNs que mesmo com o uso de glicose a $25 \%$ manifestaram dor intensa, eles estão inclusos no déficit de sucção citado anteriormente. A utilização de meio não farmacológico tem como objetivo prevenir a desorganização e agitação excessiva, reduzindo o estresse que pode intensificar a sensação de dor durante a punção. ${ }^{9}$

De acordo com os dados apresentados, observa-se, que durante a punção arterial, não foram percebidas alterações fisiológicas significativas, ressaltando que, nos casos em que os RNs se encontravam internados por um período superior a um mês, mostravam um comportamento tão condicional que só o fato de se passar o algodão com álcool para desinfecção da pele, já se mostravam desorganizados e irritados.

As pesquisas documentam que o recém-nascido tem todos os componentes funcionais e neuroquímicos necessários para a recepção e transmissão do estímulo doloroso. As exposições aos estímulos dolorosos e estressantes favorecem a desorganização fisiológica e comportamental. ${ }^{10}$ 
Tabela 3. Análise da intensidade da dor segundo solução utilizada.

Fortaleza - CE - ago/set. 2004.

\begin{tabular}{cccccc}
\hline $\begin{array}{c}\text { Medidas de sucção não } \\
\text { nutritivas }\end{array}$ & $\mathrm{N}$ & $\begin{array}{c}\text { Intensidade da dor } \\
\text { (média) }\end{array}$ & al & + & $\mathrm{D}$ \\
\hline Agua destilada & 24 & 6,08 & 23 & $-12,156$ &, 000 \\
Glicose & 24 & 1,04 & & & \\
\hline
\end{tabular}

Diante dos dados da Tabela 3, pode-se observar que houve diferença significativa na intensidade da dor entre os procedimentos realizados com a aplicação de AD e glicose $25 \%$. A intensidade da dor nos RNs é menor e suportável nos procedimentos realizados com a aplicação de glicose $25 \%$, enquanto com AD a dor é intensa. Estas diferenças são estatisticamente significantes $(p=0,00)$.

0 presente estudo mostra que a solução glicosada diminui o tempo de choro e atenuou a mímica facial de dor durante a coleta de sangue arterial. Dessa forma, acredita-se que o seu uso deva ser estimulado dentro das UTINs como medida terapêutica não farmacológica. Nossos dados corroboram outros estudos, que utilizaram medidas de sucção não nutritiva antes de realizar procedimentos dolorosos em recém-nascidos. ${ }^{11}$ Estas alterações são transitórias em RNs saudáveis, mas, no prematuro, podem causar alteração fisiológica de natureza aguda. ${ }^{12}$

A dor, quando não tratada, tem efeitos desastrosos, pois induz ao catabolismo persistente, ativa o sistema nervoso simpático, altera o sistema cardiovascular e pode desencadear ansiedade intensa e delírio. ${ }^{13,14}$

Tabela 4. Análise das alterações fisiológicas ocorridas nos recém-nascidos.

Fortaleza-CE. ago/set. 2004

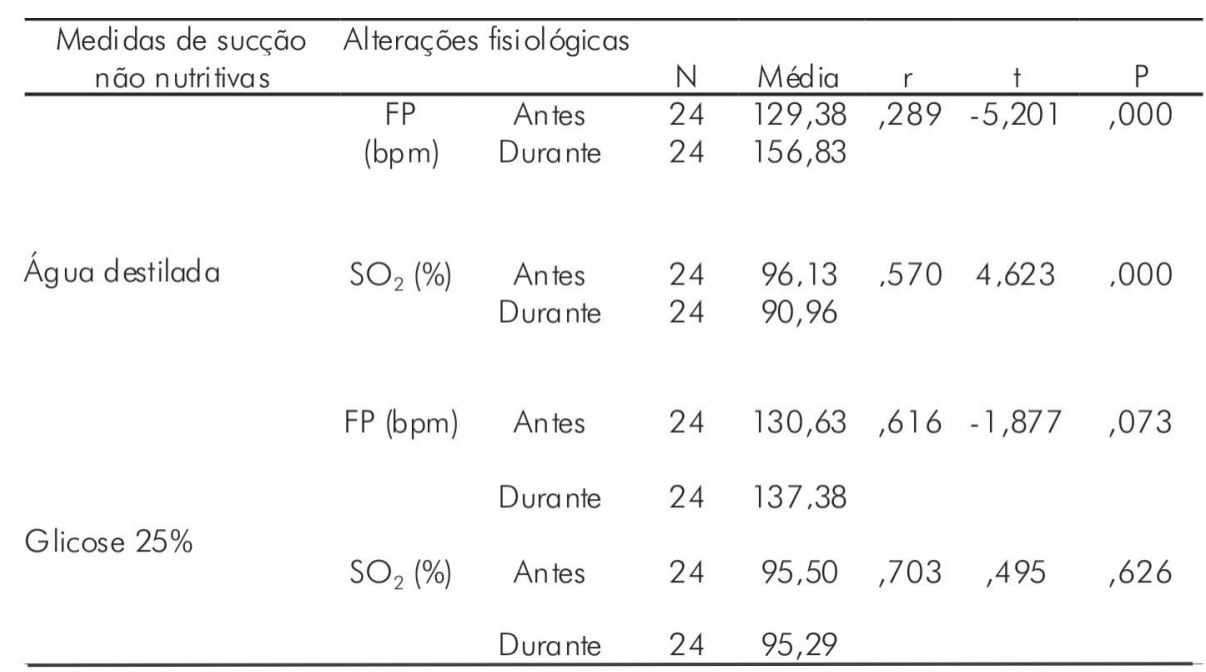

Verifica-se na Tabela 4 que houve alteração significativa tanto na frequência cardíaca quanto na saturação de oxigênio (\%) na aplicação de $A D$ antes $(p=0,00)$ e durante $(p=0,00)$ o procedimento. Não houve diferença significativa nas alterações fisiológicas com o uso da chupeta de glicose, antes $(p=0,073)$ e durante $(p=0,626)$ o procedimento.

0 uso de medidas não farmacológicas para o alívio da dor propicia maior conforto ao RN, com estabilização da FC após 0 estímulo doloroso. ${ }^{8}$

A terminação nervosa nociceptiva cutânea do RN é igual ou maior do que a de um adulto, pois $75 \%$ da transmissão se fazem por fibras não mielinizadas que são mais lentas no adulto; já no RN essas mesmas vias são mais curtas, de forma que a transmissão se faz na mesma velocidade. ${ }^{6}$ 
Tabela 5. Análise da intensidade da dor segundo a idade gestacional. Fortaleza - CE - ago/set. 2004.

\begin{tabular}{ccccccc}
\hline $\begin{array}{c}\text { Medidas de sucção não } \\
\text { nutritivas }\end{array}$ & \multicolumn{1}{l}{$\begin{array}{c}\text { Intensidade da dor } \\
\text { (média) }\end{array}$} & al & $t$ & $\mathrm{D}$ \\
\hline Água destilada & $<3$ & 1 & 28,00 & 23 & 2,26 &, 030 \\
Glicose 25\% & $>3$ & 23 & 33,26 & & &, 075 \\
& $<3$ & 21 & 33,38 & 22 & $-1,870$ & \\
\hline
\end{tabular}

Quanto à avaliação da dor segundo a idade gestacional, observou-se que, durante o procedimento com os RNs utilizando as chupetas com AD $(p=0,30)$ e com glicose $(p=0,75)$, a idade gestacional não interferiu na intensidade da dor sentida pelos RNs, nas condições estudadas na referida amostra (Tabela 5).

0 prematuro responde de maneira diferente ao estímulo doloroso. Sabe-se que, na vida fetal e neonatal, o complexo responsável pela transmissão da dor encontra-se em desenvolvimento e os mecanismos modulatórios do sistema de transmissão amadurecem mais tardiamente do que os excitatórios. ${ }^{9}$ Sendo assim, salienta-se a importância de minimizar a dor no período neonatal, pois essa fase se caracteriza como de grande fragilidade do ser humano e alta propensão a sequelas que podem resultar em quadros clínicos incapacitantes e de longa duração. ${ }^{15}$

No momento atual, a maioria dos profissionais de enfermagem consegue identificar as manifestações fisiológicas e comportamentais dos RNs. Este resultado indica uma mudança de paradigma, pois até alguns anos atrás, a informação repassada era a de que o neonato não sentia dor. ${ }^{16}$

0 adequado alívio da dor deve ser uma prioridade no planejamento da assistência, independentemente da idade bem como do tipo de procedimento a ser realizado. Cabe aos profissionais prestadores de assistência implantar estratégias que venham proporcionar o adequado alívio da dor.

\section{CONCLUSÃO}

No presente estudo permitiu-se concluir que: a glicose a $25 \%$, dois minutos antes do procedimento doloroso, permitiu ao recém-nascido ficar mais tranquilo e organizado durante 0 estímulo doloroso, e as alterações fisiológicas não variaram nos dois grupos, enquanto as comportamentais estão ligadas diretamente à intensidade da dor durante a realização dos procedimentos dolorosos.

0 uso de escalas de dor à beira do leito do RN durante procedimentos dolorosos pode vir a ser um passo fundamental para se instalarem, no Serviço de Neonatologia, medidas habituais não farmacológicas, como as chupetas de gaze embebidas em glicose a $25 \%$, pois as escalas comprovam a existência da dor e, assim, favorecerem ao RN intervenções adequadas.

\section{REFERÊNCIAS}

1. Guinsburg R. Avaliação e tratamento da dor no recém-nascido. J Pediatr 1999; 75(3): 149-60.

2. Tamez RN, Silva MJP. Enfermagem na UTI neonatal. $3^{\text {a }}$ ed. Rio de Janeiro (RJ): Guanabara Koogan; 2006.

3. Reichert APS, Silva SLF, Oliveira JM. Dor no recém-nascido: uma realidade a ser considerada. Rev Nurs 2000 nov; 30(3): 28-34.

4. Kolpeman BI, Santos AMN, Goulart AL, Almeida MFB, Miyoshi MH, Guinsburg R. Diagnóstico e tratamento em neonatologia. São Paulo (SP): Atheneu; 2004.

5. Vieira S, Hossne WS. Metodologia científica para a área de Saúde. Rio de Janeiro (RJ): Campus; 2001.

6. Segre CAM. Perinatologia: fundamentos e prática. In: Bernaldo AJN, Huberman JI. Dor no feto e no recém-nascido. São Paulo (SP): Sarvier; 2002.

7. Oliveira MMC, Leitão GCM. Refletindo sobre os cuidados aos recémnascidos de muito baixo peso em uma unidade neonatal: a importância dos conceitos. Rev Rene 2005 maio/ago; 6(2): 109-15.

8. Guinsburg R, Almeida MFB, Peres CA et al. Reliability of two behavioral tools to assess pain in preterm neonates. Med J 2003; 121 (2): 7276.

9. Gaspary LV, Rocha I. Intervenções não-farmacológicas para alívio da dor em Recém-Nascidos Prematuros (RNPT). Rev Nurs 2004; 79(7): 47-50.

10. Gaíva MAM, Dias NS. Dor no recém-nascido: percepção de profissionais de saúde de um hospital universitário. Rev Paul Enferm 2002; 21(3):155-68.

11. Stevens B, Mcgrath P, Gibbins S, Beyene J, Breau L, Camfield C, et al. Procedural pain in newborns at risk for neurologic impairment. Pain 2003; 105: 27-35.

12. Prestes AC, Guinsburg R, Balda RC, Marba ST, Rugolo LM, Pachi $P R$ et al. Freqüência do emprego de analgésicos em unidades de terapia intensiva neonatal universitárias. J Pediatr 2005; 81 (5): 40510.

13. Martin LD et al. Prospective documentation of sedative, analgesic and neuromuscular blocking agent use in infants and children in the intensive care unit: a multicenter prospective. Pediatr Crit Care Med 2001; 2:205-10. 
Dor sofrida pelo recém-nascido

Silva TM, Chaves EMC, Cardoso MVLM

14. Tobias JD. Tolerance, withdrawal, and physical dependency after long-term sedation and analgesia of children in the pediatric intensive care unit. Crit Care Med 2000; 28(21): 2122-232.

15. Santos FLB, Oliveira MIV, Bezerra MGA. Prematuridade entre recémnascidos de mães com aminiorrex prematura. Esc Anna Nery Rev Enferm 2006 dez; 10(3): 425-31.
16. Scochi CGS, Carletti M, Nunes R, Furtado MCC, Leite AM. A dor na unidade neonatal sob a perspectiva dos profissionais de enfermagem de um hospital de Ribeirão Preto-SP. Rev Bras Enferm 2006 mar/abr; 59(2): 188-94.

aExtraído da monografia de Especialização em Enfermagem Neonatológica/UFC- autoria de Silva (2004). 